\title{
Use of bioanalyzer electropherograms for quality control and target evaluation in microarray expression profiling studies of ocular tissues
}

\author{
Christina A. Harrington • Michael Winther • \\ Michelle M. Garred
}

Received: 23 October 2009 / Accepted: 9 November 2009 /Published online: 12 December 2009

(C) The Author(s) 2009. This article is published with open access at Springerlink.com

\begin{abstract}
Expression profiling with DNA microarrays has been used to examine the transcriptome of a wide spectrum of vertebrate cells and tissues. The sensitivity and accuracy of the data generated is dependent on the quality and composition of the input RNA. In this report, we examine the quality and array performance of over 200 total RNA samples extracted from ocular tissues and cells that have been processed in a microarray core laboratory over a 7year period. Total RNA integrity and cRNA target size distribution were assessed using the 2100 Bioanalyzer. We present Affymetrix GeneChip array performance metrics for different ocular samples processed according to a standard microarray assay workflow including several quality control checkpoints. Our review of ocular sample performance in the microarray assay demonstrates the value of considering tissue-specific characteristics in evaluating array data. Specifically, we show that Bioanalyzer electropherograms reveal highly abundant mRNAs in lacrimal
\end{abstract}

\author{
C. A. Harrington $\cdot$ M. Winther $\cdot$ M. M. Garred \\ Gene Microarray Shared Resource, \\ Oregon Health and Science University, \\ Portland, OR, USA \\ C. A. Harrington \\ Knight Cancer Institute, Oregon Health and Science University, \\ Portland, OR, USA \\ C. A. Harrington \\ Vaccine and Gene Therapy Institute, \\ Oregon Health and Science University, \\ Portland, OR, USA \\ C. A. Harrington $(\bowtie)$ \\ CH14G, Oregon Health and Science University, \\ 3303 SW Bond Ave, \\ Portland, OR 97239, USA \\ e-mail: harringc@ohsu.edu
}

gland targets that are correlated with variation in array assay performance. Our results provide useful benchmarks for other gene expression studies of ocular systems.

Keywords DNA microarrays · Gene expression · mRNA · RNA quality · Capillary electrophoresis · Lacrimal gland

\section{Introduction}

Expression profiling with DNA microarrays has been widely applied over the last decade to measure differences in gene expression patterns in a large variety of research samples and clinical specimens. High-density microarrays are able to survey the genome-wide transcriptome of a particular organism and present a 'snapshot' of gene expression in the cell or tissue sample at the time of RNA isolation. These profiles are generated from both homogenous and heterogeneous collections of cells and provide measures of messenger RNA (mRNA) levels from low to high abundance. The quality of the RNA samples used in these studies is crucial in generating accurate and informative results. Previous reports have described the value of determining RNA purity and integrity prior to selecting samples for microarray hybridization [1-3]. Currently, these sample attributes are commonly determined by UV spectrophotometry and RNA sizing with capillary electrophoresis on the 2100 Bioanalyzer, a microfluidics platform for electrophoretic separation of biomolecules [4]. In addition to the impact of RNA degradation on microarray analysis, we and others have observed that highly abundant hemoglobin RNA transcripts present in whole blood RNA samples can reduce overall mRNA detection sensitivity and increase sample-to-sample variation in the microarray assay [5-8]. These abundant RNA transcripts are not detectable in 
Bioanalyzer traces of total RNA, but can often be seen in the electrophorpherogram patterns of the amplified and labeled targets prepared for microarray hybridization $[6,8-10]$.

In our microarray core laboratory, we have processed over 6,000 RNA samples from human, monkey, mouse, rat, and other vertebrates. Each total RNA sample is assessed for integrity using the 2100 Bioanalyzer. RNA quality is determined by visual inspection of the electropherogram and the RNA integrity number (RIN) $[11,12]$ generated by the Bioanalyzer software program. Samples which pass core laboratory defined thresholds are converted to microarray hybridization targets and subjected to another round of quality assessment by Bioanalyzer analysis prior to application to the DNA microarray. The experimental workflow in the core laboratory is diagramed in Fig. 1. The points at which sample and data quality are inspected is illustrated to the right of the flow diagram.

Approximately 200 of the samples analyzed in the core laboratory have been extracted from ocular tissues and cell lines. In this report, we describe the use of Bioanalyzergenerated electropherograms to assess RNA quality and predict microarray assay performance of ocular tissue samples, and we demonstrate how electropherograms of labeled cRNA targets can provide insight on characteristic

experiment design, array selection, and sample prep

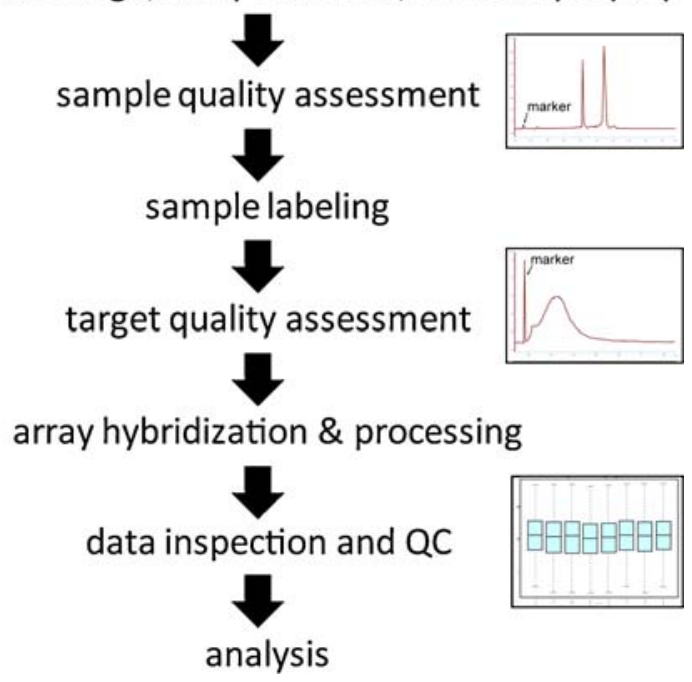

Fig. 1 Experimental workflow in the microarray core laboratory. Total RNA samples are provided to the microarray core laboratory after consultation with clients on experimental design, array selection, and sample preparation. As illustrated to the right of the flow chart, sample quality is checked prior to target synthesis, targets are assessed prior to microarray hybridization, and array data is examined for individual array performance and data distribution patterns across samples within a project. Sample, target, and array performance data are collected in a laboratory information management system for centralized information tracking and to facilitate comparisons among projects and sample types
RNA patterns associated with different tissue types. We show that while ocular RNA samples that pass total RNA quality assessment thresholds generally produce highquality microarray data, RNA from lacrimal gland and primary acinar cell cultures expresses abundant levels of a few mRNAs that may impact sensitivity and contribute to sample-to-sample variation in the microarray profiling assay.

\section{Materials and methods}

Samples

The samples analyzed in this study were submitted to the Affymetrix Microarray Core laboratory of the OHSU Gene Microarray Shared Resource over the period of 2002 to 2009. Tissues or cell cultures were harvested and RNA was isolated in the client laboratory. RNA samples were isolated using one of the following commercial kits: RNA aqueous (Ambion, Inc., Austin, TX, USA), Trizol Reagent (Invitrogen, Inc., Carlsbad, CA, USA), and RNeasy Total RNA (Qiagen, Inc., Valencia, CA, USA). Total RNA from ocular tissues and cell lines examined in this study were provided by Dr. Mitzuyoshi Azuma of the Senju Laboratory for Ocular Sciences at OHSU and Dr. Justine Smith of the Casey Eye Institute at OHSU.

\section{Bioanalyzer analysis}

Total RNA quality was assessed by determining UV 260/ 280 absorbance ratios and examining RNA size distribution on RNA Nano LabChips (Agilent Technologies, Santa Clara, CA, USA) processed on the Agilent 2100 Bioanalyzer using the total RNA electrophoresis program. For samples analyzed after 2003, a RIN was generated for each Bioanalyzer trace using 2100 Expert Software (Agilent Technologies). The maximum RIN score is 10. Labeled cRNA targets generated from the total RNA samples were examined using the mRNA program on the Bioanalyzer. There is no numerical scoring algorithm for this program.

\section{Target labeling}

All RNA samples were processed for array hybridization using the GeneChip one-cycle target labeling kit (Affymetrix, Inc, Santa Clara, CA, USA) for amplification and labeling of total RNA. Target synthesis was performed following the Affymetrix GeneChip Expression Analysis Technical Manual, rev. 5 (http://www.affymetrix.com/ support/technical/manual/expression_manual.affx) with minor modification. Using 2-4 $\mathrm{g}$ of total RNA as input, mRNA was converted to double-stranded cDNA and 
purified by phenol-chloroform-isoamyl alcohol extraction and ethanol precipitation. In a second step, amplified and biotinylated cRNA (the target) was produced by in vitro transcription. Unincorporated nucleotides were removed using the RNeasy Mini kit (Qiagen Inc., Valencia, CA, USA) followed by ethanol precipitation of the labeled target. After resuspension in RNase-free water, each target was quantified by UV260 absorbance.

\section{Microarray hybridization and processing}

Labeled targets were chemically fragmented as per Affymetrix' recommendations and combined with Affymetrix biotinylated hybridization controls (oligomer B2 and cRNAs for BioB, BioC, BioD, and $\mathrm{CreX}$ ) in hybridization buffer. Hybridizations were performed for $16 \mathrm{~h}$ at $45^{\circ} \mathrm{C}$ after addition of $10 \mu \mathrm{g}$ of target to the GeneChip array (Affymetrix) selected for each analysis. In general, speciesmatched oligonucleotide arrays were used for each target. However, microarray assays of monkey tissues before 2004 were performed on human arrays. Post-hybridization array processing of all arrays was performed according to manufacturer's recommendations for the array type.

\section{Microarray analysis and performance assessment}

The array image scan was processed with Affymetrix Microarray Suite, version 5.0 (MAS 5.0) software. All GeneChip expression arrays included in this study contain control probe sets for both spiked and endogenous RNA transcripts (e.g., BioB, BioC, BioD, CreX, and speciesspecific actin and GAPDH). Following single-array analysis of the array signal intensity pattern with MAS 5.0, six values were examined to assess overall assay performance: background, noise, average signal, \% present, ratio of signal values for probe sets representing the $5^{\prime}$ and $3^{\prime}$ ends of actin and GAPDH transcripts, and total signal for BioB, BioC, BioD, and CreX probe sets. Gene transcript levels are determined for each sample from the MAS generated .CHP files. Relative performance of groups of related samples is examined using visualization graphs of gene level data distributions, such as scatter and box plots, with Expression Console (Affymetrix) and Statistica (StatSoft, Tulsa, OK, USA).

\section{Results}

Total RNA quality assessment in the microarray core facility

The core lab received total RNA from ocular tissues, primary cultures, and cell lines collected or derived from three mammalian species: human, monkey, and rat. For each sample, RNA purity was assessed by OD 260/280 values and RNA integrity by size distribution analysis on the 2100 Bioanalyzer. All sample electropherograms are assessed for total RNA quality by visual inspection and, after 2003, by RIN score. Minimum requirements for a pass in the core laboratory are an RNA integrity value greater than 7.8 and a 260/280 ratio greater than 1.7. Of the 206 ocular RNA samples submitted, all had acceptable OD 260/ 280 values, between 1.8 and 2.2. RIN scores varied from no call to 10 ; however, the vast majority of samples received RIN scores between 8.5 and 10. Altogether, eight different ocular tissues, primary cell cultures, or cell lines were assessed. The mean RIN for each ocular cell or tissue type for which the core laboratory processed more than two samples and for which a RIN score is available is shown in Table 1; highly degraded samples with RIN scores less than 7 were excluded. Fewer than ten samples showed extensive RNA degradation (data not shown).

\section{Target cRNA pattern analysis}

In total, 181 RNA samples were converted to amplified and biotin-labeled cRNA targets for microarray hybridization. An aliquot of each cRNA target was analyzed on the 2100 Bioanalyzer. A typical size distribution profile for mammalian targets that perform well in the GeneChip microarray assay is shown in Fig. 2a. In general, each ocular tissue or cell line target demonstrated a size distribution profile consistent with high-quality total RNA samples prepared from other mammalian tissues profiled in the core laboratory (Fig. 2b, c, and d). However, targets produced from lacrimal gland RNA or acinar cells prepared from lacrimal glands showed an additional sharp peak or several smaller peaks in the profile (Fig. 2e and f). The height of this peak varied among lacrimal gland samples.
Table 1 Bioanalyzer RIN scores for total RNA samples

\begin{tabular}{lcc}
\hline Tissue/cell & No. of samples & Average RIN \\
\hline Conjunctiva primary culture & 3 & 9.9 \\
Lacrimal gland acinar cells & 19 & 9.4 \\
Retinal endothelial cells & 20 & 9.6 \\
Choroid endothelial cells & 18 & 9.6 \\
Retinal pigment epithelial cell line & 12 & 10 \\
Cornea epithelium cell line & 16 & 10 \\
\hline
\end{tabular}


Fig. 2 Bioanalyzer electropherograms of microaray targets. Electropherogram traces for approximately $200 \mathrm{ng}$ of cRNA target applied to an RNA Nano Chip were generated on the 2100 Bioanalyzer using the mRNA sizing program. a Human brain control cRNA target; $\mathbf{b}$ human cornea epithelial cells; $\mathbf{c}$ human choroid endothelial cells; d human retinal endothelial cells; e Rhesus macaque lacrimal gland acinar cells; f Rhesus macaque lacrimal gland
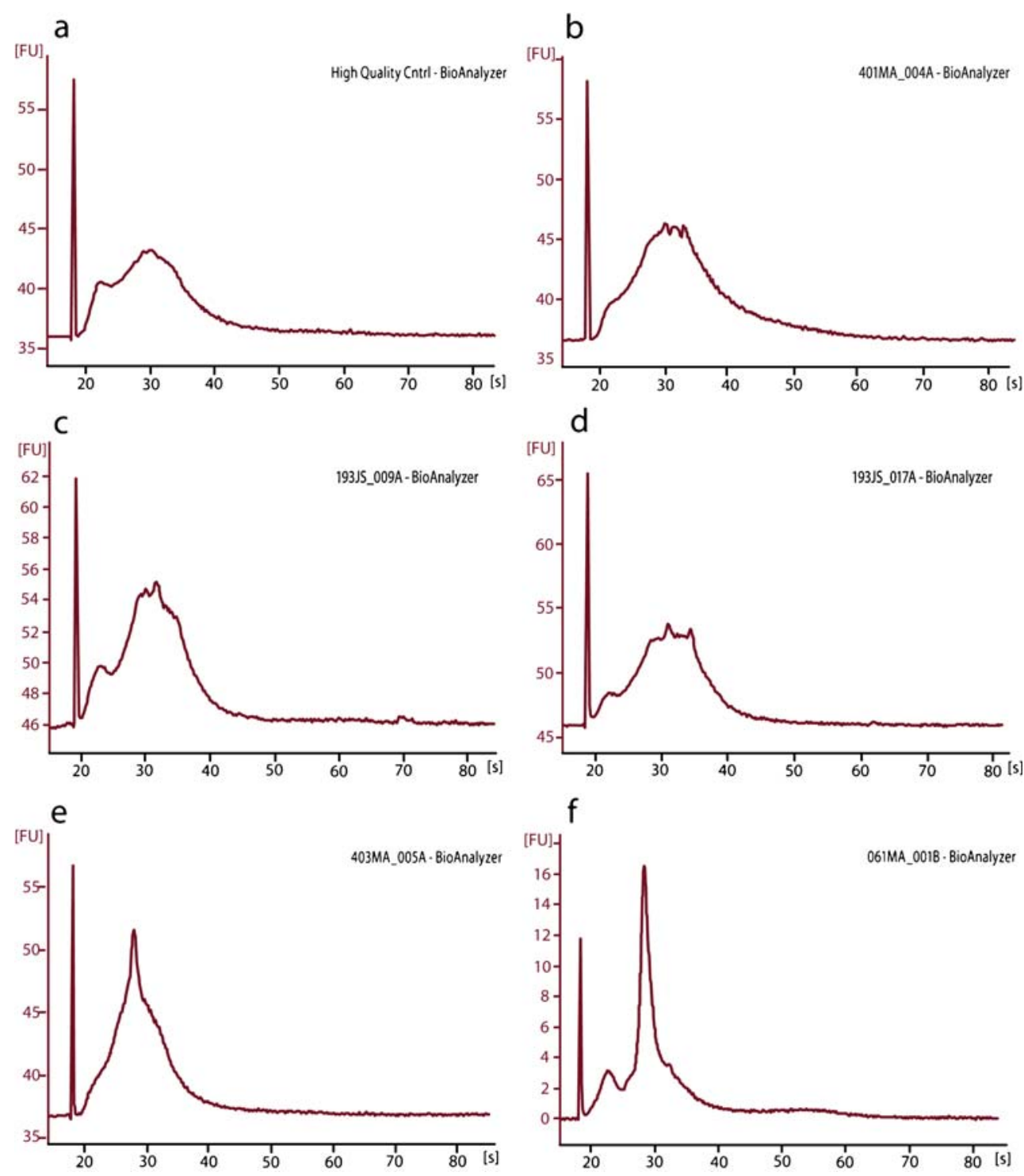

To demonstrate the effectiveness of our total RNA quality control step in excluding samples that will perform poorly in the microarray assay, we compared targets and microarray performance from a retinal endothelial sample with a subthreshold RIN score (4.3) to other high-scoring retinal endothelial samples (average RIN=9.2). The target size profile shifts to lower molecular weight RNA fragments when the low-scoring RNA was used to produce microarray target and overall target yield was threefold lower than that of the high-scoring total RNA samples.

\section{Microarray expression profiles}

All samples that passed core laboratory total RNA quality assessment performed well in the microarray hybridization assay. Typical array performance metrics are shown in Table 2 for a variety of ocular tissues and GeneChip array types. In general, samples displayed $3^{\prime} / 5^{\prime}$ ratios for the
GeneChip 'housekeeping' control genes, actin and GAPDH, of 3 or less, indicative of minimal RNA degradation prior to target labeling. Overall transcript detection, as measured by the $\%$ present $(\% \mathrm{P})$ call generated with the MAS 5.0 analysis algorithm, varied with array and tissue type. In general, performance of the ocular samples in the microarray expression assay was similar to other high-quality mammalian RNA samples profiled in the core. An exception to this performance pattern is the microarray data produced from the human retinal endothelial cell target produced from RNA with a RIN score of 4.2 . The $\% \mathrm{P}$ for this sample was only $55 \%$ compared to the population average of $60 \%$ for this tissue; the actin $3^{\prime} / 5^{\prime}$ ratio was 3.43 , two and a half times higher than the typical ratio for high-quality RNA inputs.

Acinar cell samples prepared from both rat and monkey showed good overall performance. However, there was an increased level of variation in the detection sensitivity 
Table 2 Microarray expression profiling performance metrics

\begin{tabular}{|c|c|c|c|c|c|c|}
\hline Tissue/cell & Species & Array & $\% \mathrm{P}$ & $3^{\prime} / 5^{\prime}$ actin & 3'/5' GAPDH & $n$ \\
\hline Retinal endothelial cells & Human & Human U95Av2 & 57.8 & 1.16 & 0.95 & 2 \\
\hline Retinal endothelial cells & Human & Human focus & 60.0 & 1.71 & 1.54 & 27 \\
\hline Choroid endothelial cells & Human & Human focus & 60.2 & 1.27 & 1.07 & 18 \\
\hline Retinal pigment epithelial cell line & Human & Human U133_Plus2 & 49.9 & 1.73 & 0.96 & 11 \\
\hline Cornea epithelium cell line & Human & Human U133_Plus2 & 46.7 & 1.98 & 1.03 & 16 \\
\hline Acinar cell primary culture & Monkey & Rhesus genome & 42.2 & 1.8 & 2.5 & 15 \\
\hline Conjunctiva primary culture & Monkey & Rhesus genome & 43.6 & 1.57 & 1.37 & 2 \\
\hline Lacrimal gland tissue & Monkey & Human U95Av2 & 29.1 & 5.61 & 2.72 & 2 \\
\hline Acinar cell primary culture & Rat & Rat 230_2 & 51.3 & 2.47 & 0.86 & 10 \\
\hline Lens epithelial cells & Rat & Rat U34A & 50.7 & 1.43 & 0.97 & 4 \\
\hline
\end{tabular}

metric, \% , with the nine monkey lacrimal gland acinar cell samples profiled $(40-44 \% \mathrm{P})$ compared to typical performance for other sets of similar samples in which $\% \mathrm{P}$ standard deviation is less than $1 \%$. The GeneChip Rhesus Genome array contains 52,000 probes sets designed to monitor expression of 47,000 potential transcripts [13]. Therefore, a difference of $4 \%$ in detection sensitivity corresponds to a loss of detection of about 2,000 probe sets. Figure 3 shows the monkey acinar cell target electropherograms for the samples with the lowest $\% \mathrm{P}$ value and the highest. The lower sensitivity values correspond to the samples that showed the highest peaks in the Bioanalyzer trace. The higher $\% \mathrm{P}$ values were associated with samples showing lower peaks and an overall target size distribution more similar to the majority of tissues profiled in the core.

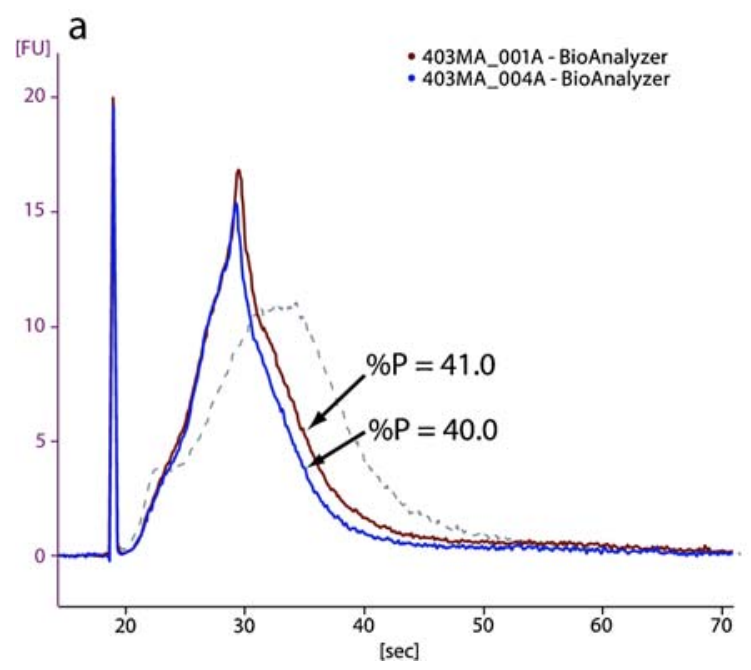

Fig. 3 Target assessment patterns of lacrimal gland samples. Electropherogram patterns for microarray targets prepared from Rhesus macaque lacrimal gland acinar cells. The gray dotted line corresponds to target prepared from high-quality human brain RNA. a Electropherogram
To gain further insight on the unusual expression profiles seen with the lacrimal gland, we determined which gene transcripts were measured at the highest levels for each sample. Transcripts for lipocalin 1, LCN1 (tear prealbumin), showed the highest hybridization signal in the monkey lacrimal gland samples. This suggests that LCN1 mRNA may correspond to the sharp peak seen in the target Bioanalyzer trace for lacrimal gland in Fig. 1f. Other abundant mRNAs associated with lacrimal gland function [14] may also contribute to the atypical peaks in the lacrimal gland and acinar cell electropherograms. Variation in genes represented on the rat and monkey arrays and limited annotation for many of the probes sets tiled on the Rhesus Genome array make it difficult at this time to identify the full complement of gene transcripts measured at the highest levels on the microarrays included in this study.

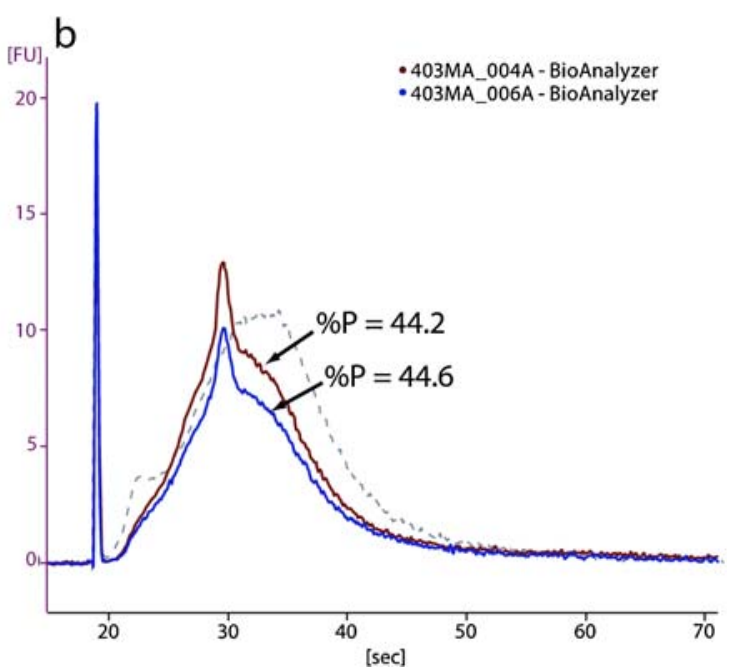

traces of the two targets associated with the lowest \%P measured in the microarray assay for group of 9 acinar cell samples processsed together; b electropherogram traces of the two targets associated with the highest $\% \mathrm{P}$ measured in the microarray assay 


\section{Discussion}

Transcriptome profiling of primary tissues, cultured cells, and immortalized cell lines is crucially dependent on the quality of the cellular RNA used for the microarray assay. Spectrophotometric measurements and molecular sizing with the 2100 Bioanalyzer have proven to be extremely useful for identifying contaminated and degraded RNA that will underperform in the microarray assay $[1,2]$. In our core laboratory, we have successfully generated microarray profiles from a variety of ocular tissues and cells by following a workflow that tests total RNA and cRNA target quality prior to array assay. Successful use of this data in expression studies of lens epithelium [15], trabecular meshwork [16], and human retinal and choroidal vascular endothelium [17] have been previously described. These studies along with reports by others on ocular tissues, such as cornea [18], mouse and rat lacrimal gland [19, 20], and rat and iris ciliary body [21], demonstrate the potential for informative expression profiling of ocular tissues and cells.

Lacrimal gland and acinar cells prepared from the lacrimal gland have an unusual target profile that is associated with an increased variation in microarray assay performance in some sample groups. The presence of discrete peaks in the lacrimal gland samples is similar to the profiles seen with whole blood RNA samples in which high levels of hemoglobin mRNAs result in one or two large, sharp peaks in the Bioanalyzer trace of targets prepared from blood RNA [6, 10]. The highly abundant hemoglobin mRNA can contribute to reduced sensitivity in the microarray expression assay and increased sample-tosample variation [5-8]. It has been suggested that increased non-specific hybridization from a few very highly expressed mRNAs can result in decreased detection of other less abundant gene transcripts [7]. Variation in the levels of high-abundance mRNAs due either to physiological differences among samples or differences in cell/tissue collection methods would then result in differences in overall signal distribution among samples that could contribute to inaccurate assessments of differential expression for some genes. The results we observe with lacrimal gland and acinar cell samples suggest that there is potential for similar problems with this tissue. Inspection of cRNA target traces prior to microarray assay can help determine whether high abundance of one or a few mRNA transcripts or a large variation in the levels of these transcripts among samples is likely to affect the microarray hybridization.

Removal of globin transcripts prior to labeling or blocking these transcripts during the reverse transcription step of the labeling procedure has been shown to produce improved microarray data from whole blood samples $[6,9,10]$. For expression profiling studies of lacrimal gland, similar approaches may be of value. High abundance and variable levels of lipocalin RNA and other highly expressed genes involved in tear production and secretion may contribute to apparent changes in transcript levels of other genes or inability to detect some mRNAs due to cross-hybridization of the abundant transcripts to probes on the array. Samples with the highest levels of a few abundant mRNAs will also tend to have the lowest measured sensitivity in the array assay. This may be incorrectly interpreted as a reduction in overall transcriptome complexity.

In conclusion, our work demonstrates that multiple ocular tissues and cell lines perform well in the microarray expression profiling assay. Routine generation of highquality data requires both standardized laboratory procedures and multiple quality control steps, as outlined in Fig. 1. Our results also show that array performance characteristics are both tissue and array specific; we provide average metrics for a variety of ocular sample types that may be used for benchmarking. Finally, we suggest that conditions or sample treatments that modify lacrimal gland secretion may lead to artifactual differences among array expression profiles due to the presence of a few highly abundant mRNA transcripts associated with the secretory function of this tissue.

Acknowledgements We thank Ryan Walkup for providing detailed information on samples provided to the core for analysis. We thank Rachel Slottke and Kristina Vartanian for technical assistance. MW and $\mathrm{CAH}$ receive support from the Knight Cancer Institute Cancer Center Support Grant, grant \# 5P30CA069533-13 and MG receives support from the Oregon Clinical and Translational Research Institute (OCTRI), grant \# UL1 RR024140 from the National Center for Research Resources (NCRR).

Open Access This article is distributed under the terms of the Creative Commons Attribution Noncommercial License which permits any noncommercial use, distribution, and reproduction in any medium, provided the original author(s) and source are credited.

\section{References}

1. Kiewe P, Gueller S, Komor M, Stroux A, Thiel E, Hofmann WK. Prediction of qualitative outcome of oligonucleotide microarray hybridization by measurement of RNA integrity using the 2100 Bioanalyzer capillary electrophoresis system. Ann Hematol. 2009;88:1177-83.

2. Jones L, Goldstein DR, Hughes G, Strand AD, Collin F, Dunnett $\mathrm{SB}$, et al. Assessment of the relationship between pre-chip and post-chip quality measures for Affymetrix GeneChip expression data. BMC Bioinformatics. 2006;7:211.

3. Strand C, Enell J, Hedenfalk I, Ferno M. RNA quality in frozen breast cancer samples and the influence on gene expression analysis - a comparison of three evaluation methods using microcapillary electrophoresis traces. BMC Mol Biol. 2007;8:38.

4. Agilent technologies application note: analysis of total RNA using the Agilent 2100 Bioanalyzer and the RNA 6000 LabChip kit. 2001 Available at: www.chem.agilent.com/Library/applications/ 59687493.pdf. 
5. Fan H, Hegde PS. The transcriptome in blood: challenges and solutions for robust expression profiling. Curr Mol Med. 2005;5 (1):3-10.

6. Vartanian K, Slottke R, Johnstone T, Casale A, Planck SR, Choi $\mathrm{D}$, et al. Gene expression profiling of whole blood: comparison of target preparation methods for accurate and reproducible microarray analysis. BMC Genomics. 2009;10:2.

7. Debey S, Schoenbeck U, Hellmich M, Gathof BS, Pillai R, Zander T, et al. Comparison of different isolation techniques prior gene expression profiling of blood derived cells: impact on physiological responses, on overall expression and the role of different cell types. Pharmacogenomics J. 2004;4(3):193-207.

8. Feezor RJ, Baker HV, Mindrinos M, Hayden D, Tannahill CL, Brownstein $\mathrm{BH}$, et al. Whole blood and leukocyte RNA isolation for gene expression analyses. Physiol Genomics. 2004;19(3):247-54.

9. Liu J, Walter E, Stenger D, Thach D. Effects of globin mRNA reduction methods on gene expression profiles from whole blood. J Mol Diagnostics. 2006;8(5):551-8.

10. Raghavachari N, Xu X, Munson PJ, Gladwin MT. Characterization of whole blood gene expression profiles as a sequel to globin mRNA reduction in patients with sickle cell disease. PLoS ONE. 2009;4(8):e6484.

11. Schroeder A, Mueller O, Stocker S, Salowsky R, Leiber M, Gassmann $\mathrm{M}$, et al. The RIN: an RNA integrity number for assigning integrity values to RNA measurements. BMC Mol Biol. 2006;7:3.

12. Agilent technologies application note: RNA integrity number (RIN)-standardization of RNA quality control. 2006. Available at: www.chem.agilent.com/Library/applications/5989-1165EN.pdf.

13. Affymetrix Data Sheet:GeneChip Rhesus Macaque Genome Array. 2005. Available at: www.affymetrix.com/support/technical/ datasheets/rhesus_datasheet.pdf.
14. Ozyildirim AM, Wistow GJ, Gao J, Wang J, Dickinson DP, Frierson HF Jr, et al. The lacrimal gland transcriptome is an unusually rich source of rare and poorly characterized gene transcripts. Invest Ophthalmol Vis Sci. 2005;46(5):1572-80.

15. Belusko PB, Nakajima T, Azuma M, Shearer TR. Expression changes in mRNAs and mitochondrial damage in lens epithelial cells with selenite. Biochim Biophys Acta. 2003;1623(2-3):13542.

16. Nakajima E, Nakajima T, Minagawa Y, Shearer TR, Azuma M. Contribution of ROCK in contraction of trabecular meshwork: proposed mechanism for regulating aqueous outflow in monkey and human eyes. J Pharm Sci. 2005;94(4):701-8.

17. Smith JR, Choi D, Chipps TJ, Pan Y, Zamora DO, Davies MH, et al. Unique gene expression profiles of donor-matched human retinal and choroidal vascular endothelial cells. Invest Ophthalmol Vis Sci. 2007;48(6):2676-84.

18. Turner HC, Budak MT, Akinci MA, Wolosin JM. Comparative analysis of human conjunctival and corneal epithelial gene expression with oligonucleotide microarrays. Invest Ophthalmol Vis Sci. 2007;48(5):2050-61.

19. Nguyen CQ, Sharma A, She JX, McIndoe RA, Peck AB. Differential gene expressions in the lacrimal gland during development and onset of keratoconjunctivitis sicca in Sjogren's syndrome (SJS)-like disease of the C57BL/6.NOD-Aec1Aec2 mouse. Exp Eye Res. 2009;88(3):398-409.

20. Nguyen DH, Toshida H, Schurr J, Beuerman RW. Microarray analysis of the rat lacrimal gland following the loss of parasympathetic control of secretion. Physiol Genomics. 2004;18(1):10818.

21. Ohta K, Kikuchi T, Miyahara T, Yoshimura N. DNA microarray analysis of gene expression in iris and ciliary body of rat eyes with endotoxin-induced uveitis. Exp Eye Res. 2005;80(3):401-12. 